Gynäkologische Endokrinologie

2009. 7:217-218

DOI 10.1007/s10304-009-0336-3

Online publiziert: 4. Oktober 2009

(c) Springer Medizin Verlag 2009

\author{
A. Schröer ${ }^{1} \cdot$ M. Ludwig ${ }^{2} \cdot$ K. Diedrich ${ }^{1}$ \\ ${ }^{1}$ Klinik für Frauenheilkunde und Geburtshilfe, USKH Campus Lübeck \\ ${ }^{2}$ ENDOKRINOLOGIKUM Hamburg, Hamburg
}

\title{
Schilddrüsenerkrankungen in der Frauenheilkunde und Geburtshilfe
}

Schwangerschaft bedarf es zunächst eines eingehenden Verständnisses der physiologischen hormonellen Veränderungen sowie einer exakten Kenntnis der relevanten Referenzwerte, um korrekte Diagnosen und Therapien bei Störungen erstellen zu können. Für die Praxis ist es aufgrund drohender Einschränkungen der intellektuellen Entwicklung des Kindes essenziell, Jodmangel und eine Hypothyreose vor und in der Schwangerschaft rechtzeitig zu erkennen und zu therapieren. Mit diesen Aspekten befasst sich der erste Beitrag dieses Heftes (Dawson et al.). dings noch eher eine untergeordnete Rolle, und es herrscht vielfach große Unsicherheit darüber, welche Untersuchungen zu welcher Zeit sinnvoll und angemessen sind und mit welchen Erkrankungen bei der Betreuung von Frauen und Schwangeren typischerweise gerechnet muss. Darüber hinaus erfordert die Diagnostik und adäquate Therapie von Schilddrüsenerkrankungen in der Frauenheilkunde und Geburtshilfe ein interdisziplinäres und koordiniertes Vorgehen zwischen Frauenärzten, Internisten, Endokrinologen und Kinderärzten. Wir möchten mit diesem Heft dazu beitragen, einige wichtige und praxisnahe Aspekte rund um die Schilddrüse in der Gynäkologie und Geburtshilfe herauszustellen, um die Betreuung und Behandlung von Frauen mit Schilddrüsenstörungen zu erleichtern.

Aufgrund der möglichen schweren Störungen der Schilddrüsenfunktion und der schwerwiegenden Folgen für die Mutter und das ungeborene Kind liegt ein Schwerpunkt dieses Heftes auf Schilddrüsenerkrankungen in der Schwangerschaft und Stillzeit. Zur Erkennung von Schilddrüsendysfunktionen während der
D Störungen der Schilddrüsenfunktion können schwerwiegenden Folgen für die Mutter und das ungeborene Kind haben

Die rechtzeitige Erkennung und adäquate Therapie von Schilddrüsenentzündungen in einer Schwangerschaft ist Thema des Beitrags von Harbeck et al. Diese Erkrankungen sind von besonderer Bedeutung, da eine nicht erkannte oder falsch behandelte Thyreoiditis einen negativen Einfluss auf den Verlauf der Schwangerschaft entinnen muss auch mit einer erhöhten Wahrscheinlichkeit für eine PostpartumThyreoiditis gerechnet werden, die unterschiedliche Verläufe nehmen kann und verschiedene weitere medizinische Probleme mit sich bringen kann. Aus therapeutischer Sicht ist insbesondere die Abgrenzung von einer Hyperthyreose im Rahmen eines M. Basedow oder eines autonomen Adenoms wichtig, da diese Erkrankungen mit Thyreostatika behandelt werden. Im Gegensatz dazu kommen bei einer transienten Hyperthyreose im Rahhaben kann. Bei bestimmten Risikopati- men einer Thyreoiditis symptomatisch $\beta$ Blocker zum Einsatz.

Nicht erst in der Schwangerschaft und Stillzeit sollten eine angemessene Diagnostik der Schilddrüsenfunktion und eine sinnvolle Therapie von Dysfunktionen erfolgen. Nicht selten spielt eine Schilddrüsenfunktionsstörung zumindest anteilig bei unerfülltem Kinderwunsch eine Rolle. Durch die rasante Fortentwicklung der Behandlungsmöglichkeiten im Bereich der Reproduktionsmedizin rückt dieses Thema zunehmend ins Zentrum der täglichen Praxis. Im Beitrag von Beyer und Schultze-Mosgau wird eine adäquate Schilddrüsendiagnostik mit den konsekutiven Therapieansätzen erklärt.

Nicht zuletzt möchten wir aber auch einen allgemeinen Einblick in Schilddrüsenerkrankungen geben. Mit diesen Aspekten befassen sich die Beiträge von Janßen und Benker sowie Cordes et. al. Dies ist nicht zuletzt deshalb angebracht, da diese Erkrankungen überaus häufig sind: In Deutschland findet sich bei jedem dritten Erwachsenen eine Struma oder Struma nodosa. Funktionsstörungen der Schilddrüse einschließlich ihrer latenten Formen finden sich bei bis zu 10\% der Bevölkerung. Anzeichen von Schilddrüsenerkrankungen sind vielfältig und können von symptomlosen Zuständen bis hin zu hypertensiven Krisen oder schweren Depressionen reichen. Schilddrüsenfunktionsstörungen sind darüber hinaus in verschiedenen Bereichen der Gynäkologie von Bedeutung. Diese reichen von leichten Zyklusstörungen über schwere Fertilitätsstörungen bis hin zu einer erhöhten Abortneigung. Zusätzlich gibt es enge Verbindungen der Schilddrüsenfunktionsstörungen und anderen Erkrankun- 
gen. Ziel der strukturierten und interdisziplinären Untersuchung ist die Diagnosestellung der zugrunde liegenden Schilddrüsenerkrankung und die Bestimmung der Therapieindikation. Dazu werden morphologische Veränderungen, Funktionsstörungen, Hinweise für eine Autoimmunerkrankung oder Entzündung und das Vorhandensein von Begleiterkrankungen erfasst.

Die Herausgeber hoffen, dem Leser mit diesem Schwerpunktheft eine fundierte Grundlage für das verantwortungsbewusste Handeln bei der Diagnostik und Therapie von Schilddrüsenerkrankungen an die Hand geben zu können.

\section{Andreas filtoes}

\author{
A. Schröer
}

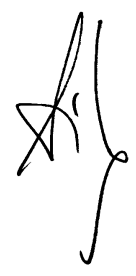

M. Ludwig

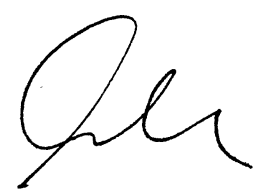

K. Diedrich

\section{Korrespondenzadresse}

\section{Dr. A.Schröer}

Klinik für Frauenheilkunde und Geburtshilfe, USKH Campus Lübeck Ratzeburger Allee 160, 23538 Lübeck andreas@drschroer.de

\section{Themenübersicht}

Die „Gynäkologische Endokrinologie“ bietet Ihnen umfassende und aktuelle Beiträge zu interessanten Themenschwerpunkten aus allen Bereichen der gynäkologischen Endokrinologie und Reproduktionsmedizin.

Wir haben die Jahrgänge 2008/2009 im Überblick für Sie zusammengestellt:

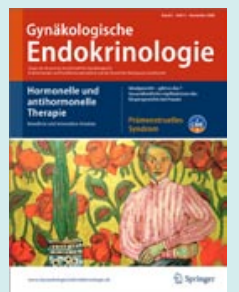

2008

- Heft 1/08 Adipositas

- Heft 2/08 Komplementärmedizin

- Heft 3/08 Die Lutealphase

- Heft 4/08 Update hormonelle Therapie

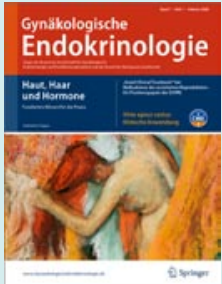

2009

- Heft 1/09 Haut, Haar und Hormone

- Heft 2/09 Assistierte Reproduktion: Bessere Ergebnisse durch ergänzende Therapieoptionen?

- Heft 3/09 Störungen der Geschlechtsidentität

- Heft 4/09 Schilddrüse

Bestellen Sie einzelne Ausgaben oder abonnieren Sie die Zeitschrift zum Preis von EUR 119,pro Jahr (zzgl. Versandkosten, Ermäßigung für Ärzte in Aus- und Weiterbildung)! Unser Kundenservice steht Ihnen für Fragen und Informationen gerne zur Verfügung:

Springer-Verlag, Kundenservice Zeitschriften

Haberstr. 7, 69126 Heidelberg

Tel.: +49 6221-345-4303, Fax: +49 6221-345-4229

E-Mail: subscriptions@springer.com

\section{Beiträge auf einen Blick}

Abonennten haben online Zugriff auf alle Beiträge im elektronischen Volltextarchiv unter www.GynaekologischeEndokrinologie.de.

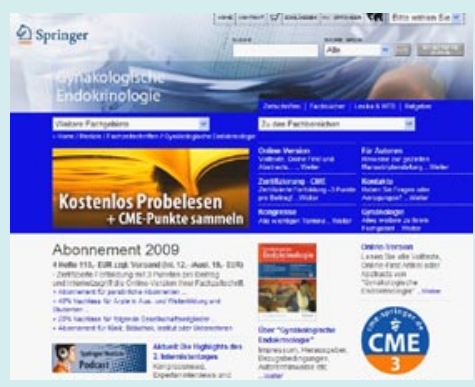

Ins Volltextarchiv gelangen Sie von der Startseite über den Navigationspunkt "Online Version".

Nach einer einmaligen Registrierung können Sie die Beiträge als pdf oder in der html-Version abrufen. 